\title{
Study of 10M' Nanotwinned Phase in the Vicinity of Martensitic Transformation in Ni-Mn-Ga Magnetic Shape Memory Alloy
}

\author{
P. VeřtáT ${ }^{a, b, *}$, L. StrakA ${ }^{a, c}$, J. DrahokOUPIL ${ }^{a}$ AND O. HeCZKo ${ }^{a, c}$ \\ ${ }^{a}$ Institute of Physics of the Czech Academy of Sciences, Na Slovance 2, 18221 Praha 8, Czech Republic \\ ${ }^{b}$ Czech Technical University in Prague, Faculty of Nuclear Sciences and Physical Engineering, \\ Department of Solid State Engineering, Trojanova 13, 12000 Prague, Czech Republic \\ ${ }^{c}$ Charles University, Faculty of Mathematics and Physics, Ke Karlovu 5, 12116 Prague, Czech Republic
}

$\mathrm{Ni}_{50.0} \mathrm{Mn}_{28.5} \mathrm{Ga}_{21.5}$ single crystal exhibiting magnetic shape memory effect was analysed by X-ray diffraction, electric resistivity and differential scanning calorimetry. Based on the intensities of the measured diffraction peaks, we discuss the evolution of the structure and microstructure during heating and cooling. Electric resistivity and DSC curves detect anomalous behaviour prior the transformation from martensite to austenite. We ascribe these pre-transformation changes in measured curves to formation of the recently discovered nanotwinned form of $10 \mathrm{M}$ martensite.

DOI: 10.12693/APhysPolA.134.859

PACS/topics: 61.05.cp, 61.50.-f, 75.50.Cc, 75.80.+q

\section{Introduction}

The discovery of magnetic shape memory (MSM) effect by Ullakko et al. in 1996 [1] started a development of MSM alloys with exceptional properties for applications as sensors and actuators [2-4]. The archetypal and probably most studied MSM alloy is the $\mathrm{Ni}^{-}$ $\mathrm{Mn}-\mathrm{Ga}$. Martensite of $\mathrm{Ni}-\mathrm{Mn}-\mathrm{Ga}$ exhibits giant magnetic field-induced strain (up to $12 \%$ [5]) in a moderate magnetic field $(<1 \mathrm{~T})$. This strain originates from the reorientation of the structural martensitic twin variants caused by twin boundary motion. Thus, it strongly depends on the twinned microstructure and high mobility of the twin boundaries [6]. The twinned microstructure of MSM alloys originates from the martensitic transformation from the cubic austenite phase to lower symmetry martensite. We focus on the non-stoichiometric compositions of the Ni-Mn-Ga resulting in five-layered modulated monoclinic martensite structure $(10 \mathrm{M})$, which is the most promising structure for applications [3, 4].

Using X-ray diffraction, we discovered recently that a new intermediate phase can form between ordinary $10 \mathrm{M}$ martensite and austenite [7]. In alloy of specific composition few tenths of kelvin below the martensitic transformation, the five-layered modulated monoclinic martensite (10M) changed to an orthorhombic intermediate (10M') phase. The SEM observations, XRD measurements and theoretical calculations indicated that such an intermediate phase could be actually the nanotwinned $10 \mathrm{M}$ martensite, with the nanotwinning suggested to be resulting from the complex hierarchical branching on the austenite nucleus - martensite interface [7].

\footnotetext{
* corresponding author; e-mail: vertat@fzu.cz
}

To obtain detailed insight to this newly discovered nanotwinned microstructure we further analyse measured XRD data. The measurements of electric resistivity and differential scanning calorimetry reveal more details on the evolution of the new phase with temperature. Moreover, from the calorimetry we estimate $a / b$ twin boundary energy of $10 \mathrm{M}$ martensite.

\section{Experimental arrangement}

We investigated $\mathrm{Ni}_{50.0} \mathrm{Mn}_{28.5} \mathrm{Ga}_{21.5}$ (in at. \%) single crystals produced by AdaptaMat Ltd. Cuboid samples of dimensions $0.9 \times 2.4 \times 20 \mathrm{~mm}^{3}$ and $0.9 \times 2.4 \times 5 \mathrm{~mm}^{3}$ were cut with faces approximately along $\{100\}$ plane of cubic austenite. Transformation from austenite to martensite occurred at $\approx 327 \mathrm{~K}$, reverse transformation temperature was $\approx 333 \mathrm{~K}$. At room temperature, the samples had $10 \mathrm{M}$ modulated martensite structure with lattice parameters $a=0.5974 \mathrm{~nm}, b=0.5949 \mathrm{~nm}, c=0.5589 \mathrm{~nm}$, and $\gamma=90.34^{\circ}$.

The XRD measurements were done on PANalytical X'Pert PRO diffractometer in Bragg-Brentano geometry with Co $(\lambda=0.1789 \mathrm{~nm})$ tube. Samples were attached to a Peltier module for heating and put to the ATC-3 texture cradle used as goniometer enabling rotation, inclination and horizontal motion of the sample. Line focus with divergent slits equal to $1^{\circ}$ was used for continuous monitoring of the reflections while heating or cooling down.

For reflections fitting, a custom-made program based on MS Excel and VBA for applications was used together with the solver.xlam add-in for the least squares method implementation. Diffraction profiles were fitted with pseudo-Voigt function defined by four parameters intensity at maximum, position in $2 \theta$, width of the peak and shape parameter (ratio of the Cauchy and Gaussian contribution). Program allows for fitting up to three overlapped peaks and independent fixing of the parame- 
ters in problematic cases or for using the average value of several fits where no evolution in some of the parameters is expected. Integral intensity of reflections was calculated using the analytic way from the parameters of the fitting function.

Resistivity of the samples was measured by the AC four-point method $(f=11 \mathrm{~Hz})$. Stanford Research Systems SR830 DSP Lock-In amplifier was used for controlling the input current from the Pico Precision PP102 current source. The output signal was amplified by PP560 low noise amplifier and detected by the same SR830. Simple filament-based heater was used for heating the samples during resistivity measurements. For temperature sensing, Keithley 195A digital multimeter and $\mathrm{Pt}$ resistance thermometer was used.

Differential scanning calorimetry was carried out with Linkam DSC6000 system equipped with the LNP95 cooling system. Three rounds of calorimetric measurements were done with the heating/cooling rate of $5 \mathrm{~K} / \mathrm{min}$.

\section{Results and discussion}

The formation of the nanotwinned 10M' phase was initially described by Straka et al. [7]. Here, based on detailed temperature measurements of the diffraction patterns, we further discuss the evolution and nature of the newly-observed phase.

We continuously measured 2Theta-Omega scans in the angular range $72^{\circ}-76^{\circ}$, in which 400 and 040 reflections of $10 \mathrm{M}$ martensite were present and where possible austenite 400 peak could be expected. Samples were reoriented in a way that the $a$ and $b$ axes were perpendicular to the studied surface prior the measurements [8]. Due to the use of the divergent beam geometry and due to the twinned structure, we were able to observe both $400_{10 M}$ and $040_{10 M}$ reflections in about $20 \mathrm{~s}$ lasting scan. This allowed for sensitive observation of temperature evolution when heating and cooling slowly (rate $0.8 \mathrm{~K} / \mathrm{min}$ or lower).

Diffraction patterns at three selected temperatures are depicted in Fig. 1. In lower temperature region (305$325 \mathrm{~K}$ ), two major reflections $400_{10 M}$ and $040_{10 M}$ were present. Although fitting the pattern with only two reflections gives a good agreement with measured data, including the third reflection, corresponding to the nanotwinned phase, leads to a better fit. This shows that the nanotwinned phase might be partially present even at low temperatures. Fitting with symmetrical pseudoVoigt function gave good agreement with measured data, no asymmetry corrections were necessary. This is also apparent from the crystallographic $R_{W P}$ factor $=0.078$.

When heating, the positions of $400_{10 M}$ and $040_{10 M}$ reflections shift continuously with no anomalous effects (see Fig. 2a), until approx. $326 \mathrm{~K}$ (Fig. 1b), where 400 $10 M^{\prime}$ reflection of the new phase becomes distinct. Observed pattern cannot be fitted using $400_{10 M}$ and $040_{10 M}$ reflections only, even when using asymmetric fitting functions. Depicted fit using three symmetric pseudo-Voigt functions has $R_{W P}=0.076$.

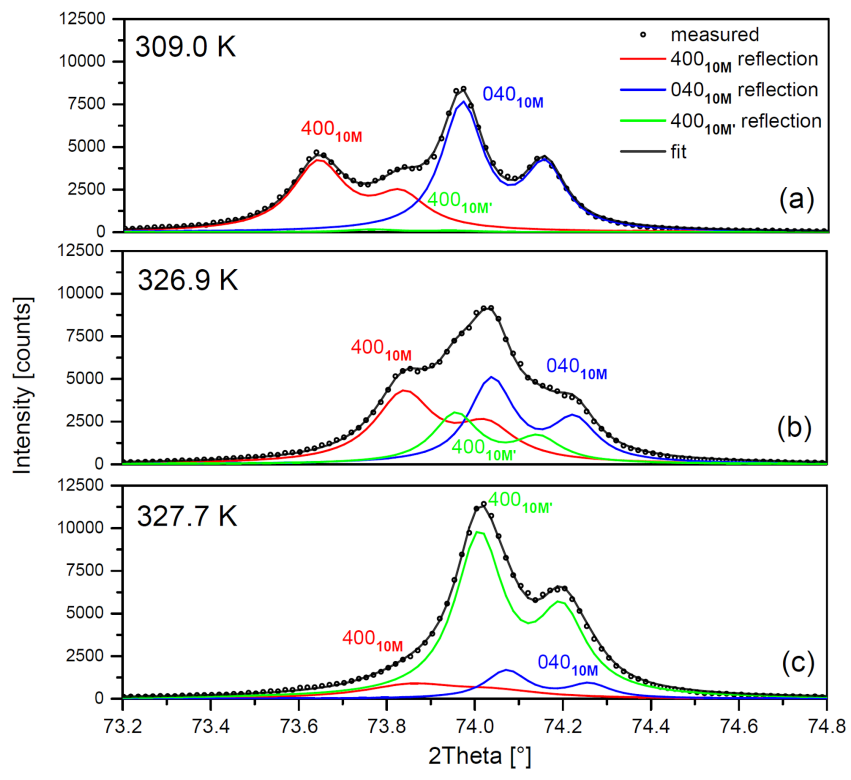

Fig. 1. Diffraction profiles measured at three selected temperatures. (a) At $309 \mathrm{~K}$, two major reflections $400_{10 M}$ and $040_{10 M}$ are observable due to use of the divergent geometry and the twinned microstructure. Including another reflection $400_{10 M^{\prime}}$ led to a better fit. (b) At $326.9 \mathrm{~K}$ noticeable changes occur, the intensity of the $400_{10 M^{\prime}}$ reflection increases significantly. (c) At $327.7 \mathrm{~K}$, the $400_{10 M^{\prime}}$ reflection is dominant while the $400_{10 M}$ and $040_{10 M}$ reflections are still present.

Finally, at $327.7 \mathrm{~K}$ (Fig. 1c) the reflection $400_{10 M^{\prime}}$ corresponding to the nanotwinned phase is dominant, while $400_{10 M}$ and $040_{10 M}$ have lost the majority of their intensity. The fit is still very good with $R_{W P}=0.081$.

As the low intensity reflections are present, some parameters (especially shape and width parameter) had to be fixed during fitting on some temperature intervals, where no development of these was expected. Prior to this, multiple fits without any restrictions and with different initialisation parameters were performed in order to obtain an insight into the tendencies, certainty and average values to be used in the final fit. This secured better stability and prevented collapsing to the local minima while fitting.

Final integral intensities of the observed reflections obtained from the fit are shown in Fig. 2. For completeness, the lattice parameters already published and discussed in [7] are also depicted.

Ratio of the $a$ and $b$ intensities of the original a/b laminate slightly changes during the heating up to $326 \mathrm{~K}$. This effect might also be caused by the constant inclination angle used during the whole measurement - one reflection can get slightly more into "focus" while the other one might slightly get out during heating.

Linear increase of the overall intensity in the low temperature region might by ascribed to slight changes in geometry due to thermal expansion of the sample. As the background might change during the measurement, 

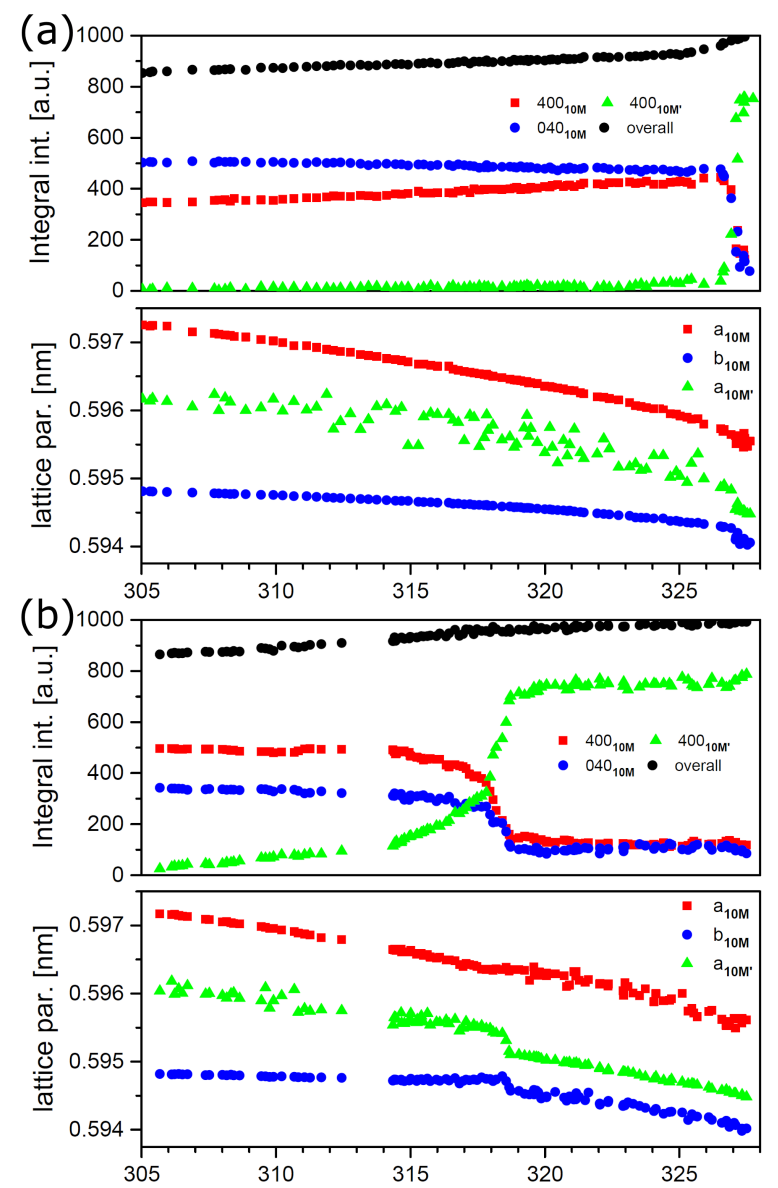

Fig. 2. Integral intensities of the observed reflections during heating (a) and cooling (b). Sum of all three intensities is also depicted. For illustration, the calculated lattice parameters obtained from the same reflections published in [7] are also shown.

this can further affect precise evaluation of the integral intensity value. When the nanotwinning becomes significant, the overall intensity increases even more. This could be ascribed to the change of microstructure.

Heating was stopped before the occurrence of austenite and no austenite peak was present in the measured scans. Due to divergent beam geometry, such a peak would appear at $\sim 75.5^{\circ}$ (region not shown in Fig. 1 but measured). If the austenite peak appeared, the overall integral intensity of the $400_{10 M}, 040_{10 M}$ and $400_{10 M^{\prime}}$ reflections would decrease. However, as the overall intensity is increasing, not decreasing, we can assume no austenite nucleus forms within irradiated region.

During cooling, hysteresis behaviour occurs. The $400_{10 M^{\prime}}$ reflection is dominant until cooling down to $318 \mathrm{~K}$, where $400_{10 M}$ and $040_{10 M}$ regain their intensity. According to our results, the nanotwinned phase is partially present even at the end of the cooling process. The high intensities of the $400_{10 M}$ and $040_{10 M}$ peaks indicate that the majority of the sample returned to the original spontaneously created $a / b$ laminate of the $10 \mathrm{M}$ martensite at the end of cooling.
To further study the transformation processes, we measured the temperature evolution of the electric resistivity. During heating, after the nearly linear change from the room temperature, the large and sharp decrease in resistivity detected is caused by the martensitic transformation [9]. The different resistivity of martensite is partially caused by the different cross-section of the sample (difference less than 3\%). However, the main reason for the higher resistivity of martensite can be the interaction of carriers with twin microstructure [10] and changes in electronic structure including Fermi surface nesting [11].

Detail look reveals the pre-transformation changes in the temperature dependence of electric resistivity, just few tenths of kelvin below the transformation to austenite (Fig. 3). This behaviour strongly correlates with the presence of the nanotwinned 10M' phase described above. The small change in the resistivity (approx. $6.3 \%$ of the entire resistivity change) could have been caused by transformation to a new phase or rather by changes in twinned microstructure. Refinement of the $a / b$ laminate resulting in channelling of the conductivity along the twin boundaries could be speculated. During cooling from austenite, no anomalous effect was observed (not shown).

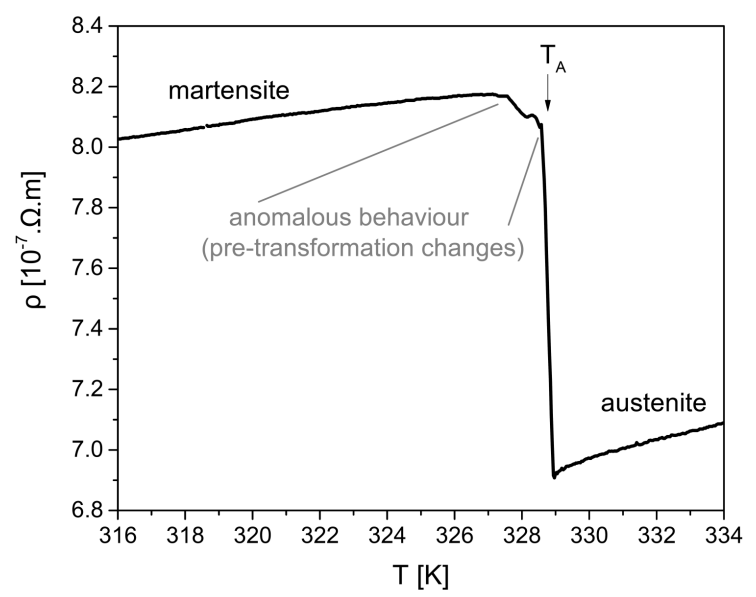

Fig. 3. Electric resistivity as a function of temperature. Nearly linear evolution during heating in martensite is followed by an anomalous behaviour before the steep jump to the austenite at $T_{A}$.

Similar pre-transformation behaviour as that of the electric resistivity was found by DSC (Fig. 4). In this case, some minor change was detected $4 \mathrm{~K}$ below the $T_{A}$ temperature, followed by a large peak due to martensitic transformation, which is first order transformation. The transformation to austenite was very sharp, in agreement with resistivity measurement. Repeated behaviour was detected in three rounds of measurements, as shown in the inset.

The anomalous behaviour represents $5.6 \%$ of the overall heat consumed during the entire transformation. The latent heat of the transformation is about $11 \mathrm{~J} / \mathrm{g}$. If only the formation of additional $a / b$ twin boundaries is considered, then their surface energy can be calculated from 


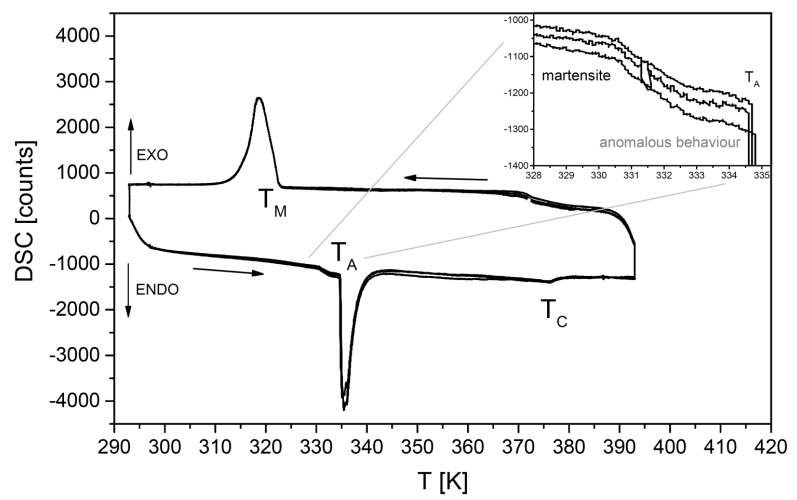

Fig. 4. DSC curves from the three rounds of measurements. Peaks correspond to the consumption or the generation of the heat during the martensitic transformation. Transformation temperatures from martensite to austenite $\left(T_{A}\right)$, from austenite to martensite $\left(T_{M}\right)$ and the Currie temperature $\left(T_{C}\right)$ are marked. In the inset, the detail of the DSC curves during heating close to the martensitic transformation is depicted. Linear evolution during heating in martensite is followed by the pre-transformation changes before the steep change indicating the martensitic transformation at $T_{A}$.

the anomalous heat. This gives the surface energy density of $a / b$ twin boundaries $\approx 3 \mathrm{meV} / \AA^{2}$ for assumed twin width $10 \mathrm{~nm}$. Considering the model [7], we can assume that some heat is consumed on the formation of austenite nuclei and therefore the true surface energy of the twin boundaries may be actually much lower. The theoretical ab-initio calculation gives the $a / b$ twin boundary energy of $0.1 \mathrm{meV} / \AA^{2}$ [7] and Kaufmann et al. calculated for the $a / c$ twin boundary of non-modulated martensite the value of $2 \mathrm{meV} / \AA^{2}[12]$.

The differences in temperatures determined by DSC and resistivity can be ascribed to the different heating rate, different experimental arrangement and the different sample used. Even though the samples were cut from the same single crystalline ingot, it is common that the composition along the ingot differs slightly along the axis [13]. This can explain the observed difference as the properties of off-stoichiometric $\mathrm{Ni}-\mathrm{Mn}-\mathrm{Ga}$ alloys are highly dependent on composition $[14,15]$. However, the overall effect of anomalous changes before the martensitic transformation is clearly noticeable in both cases indicating new 10M' phase which was identified by X-ray diffraction.

\section{Conclusion}

We studied structural changes in $\mathrm{Ni}_{50.0} \mathrm{Mn}_{28.5} \mathrm{Ga}_{21.5}$ single crystal by means of X-ray diffraction, electric resistivity and DSC measurements to characterize 10M' phase occurring in the vicinity of martensitic transformation. The new 10M' phase is formed by nanotwinning of modulated 10M martensitic phase. The process of nanotwinning is expressed as anomalies in resistivity and DSC curves. The integral intensities of X-ray lines indicate that the 10M' nanotwinned phase might be partially present even at room temperature. From anomalous heat changes measured by DSC we estimate the upper bound of $a / b$ twin boundary energy to be $3 \mathrm{meV} / \AA^{2}$.

\section{Acknowledgments}

This work was supported by the Czech Science Foundation [grant numbers 14-36566G (J. D.) and 1700062S], Ministry of Education, Youth and Sports of the Czech Republic [CZ.02.1.01/0.0/0.0/15_003/0000487] and Czech Technical University in Prague [grant number SGS16/245/OHK4/3T/14]. Authors would like to thank S. Sedláková for performing DSC measurements.

\section{References}

[1] K. Ullakko, J. K. Huang, C. Kanter, V.V. Kokorin, R.C. O'Handley, Appl.Phys.Lett. 69, 1966 (1996).

[2] S.A. Wilson, R.P.J. Jourdain, Q. Zhang, R.A. Dorey, C.R. Bowen, M. Willander, Q.U. Wahab, M. Willander, M.A.H. Safaa, O. Nur, E. Quandt, C. Johansson, E. Pagounis, M. Kohl, J. Matovic, B. Samel, W. van der Wijngaart, E.W.H. Jager, D. Carlsson, Z. Djinovic, M. Wegener, C. Moldovan, R. Iosub, E. Abad, M. Wendlandt, C. Rusu, K. Persson, Mat. Sci. Eng. R. Rep. 56, 1 (2007).

[3] A.R. Smith, A. Saren, J. Jarvinen, K. Ullakko, Microfluid. Nanofluidics 18, 1255 (2015).

[4] M. Kohl, M. Gueltig, V. Pinneker, R. Yin, F. Wendler, B. Krevet, Micromachines 5, 1135 (2014).

[5] A. Sozinov, N. Lanska, A. Soroka, W. Zou, Appl.Phys.Lett. 102, 021902 (2013).

[6] O. Heczko, Mat Sci Tech. 30, 1559 (2014).

[7] L. Straka, J. Drahokoupil, P. Vertat, J. Kopecek, M. Zeleny, H. Seiner, O. Heczko, Acta Mater. 132, 335 (2017).

[8] O. Heczko, N. Scheerbaum, O. Gutfleisch, in: Nanoscale Magnetic Materials and Applications, Eds. J.P. Liu, E. Fullerton, O. Gutfleisch, D.J. Sellmyer, Springer US, 2009, p. 399.

[9] P. Veřtát, J. Drahokoupil, O. Perevertov, O. Heczko, Phase Transit. 89, 752 (2016).

[10] J. Seidel, G. Singh-Bhalla, Q. He, S.-Y. Yang, Y.H. Chu, R. Ramesh, Phase Transit. 86, 53 (2013).

[11] P. Entel, M.E. Gruner, A. Dannenberg, M. Siewert, S.N. Nayak, H.C. Herper, V. Buchelnikov, Mater Sci Forum. 635, 3 (2010).

[12] S. Kaufmann, U.K. Rößler, O. Heczko, M. Wuttig, J. Buschbeck, L. Schultz, S. Fähler, Phys. Rev. Lett. 104, 145702 (2010).

[13] D. Kellis, A. Smith, K. Ullakko, P. Müllner, J. Cryst. Growth 359, 64 (2012).

[14] V.A. Chernenko, Scr. Mater. 40, 523 (1999).

[15] N. Lanska, O. Söderberg, A. Sozinov, Y. Ge, K. Ullakko, V.K. Lindroos, J. Appl. Phys. 95, 8074 (2004). 